\title{
Video Article \\ Using Insect Electroantennogram Sensors on Autonomous Robots for Olfactory Searches
}

\author{
Dominique Martinez ${ }^{1}$, Lotfi Arhidi ${ }^{1}$, Elodie Demondion ${ }^{2}$, Jean-Baptiste Masson ${ }^{3}$, Philippe Lucas ${ }^{2}$ \\ ${ }^{1}$ UMR 7503, Laboratoire Lorrain de Recherche en Informatique et ses Applications (LORIA), Centre National de la Recherche Scientifique (CNRS) \\ ${ }^{2}$ UMR 1392 iEES-Paris, Institut d'Ecologie et des Sciences de l'Environnement de Paris \\ ${ }^{3}$ Physics of Biological Systems, Institut Pasteur
}

Correspondence to: Dominique Martinez at Dominique.Martinez@loria.fr

URL: https://www.jove.com/video/51704

DOI: doi:10.3791/51704

Keywords: Neuroscience, Issue 90, robotics, electroantennogram, EAG, gas sensor, electronic nose, olfactory search, surge and casting, moth, insect, olfaction, neuron

Date Published: 8/4/2014

Citation: Martinez, D., Arhidi, L., Demondion, E., Masson, J.B., Lucas, P. Using Insect Electroantennogram Sensors on Autonomous Robots for Olfactory Searches. J. Vis. Exp. (90), e51704, doi:10.3791/51704 (2014).

\section{Abstract}

Robots designed to track chemical leaks in hazardous industrial facilities ${ }^{1}$ or explosive traces in landmine fields ${ }^{2}$ face the same problem as insects foraging for food or searching for mates ${ }^{3}$ : the olfactory search is constrained by the physics of turbulent transport ${ }^{4}$. The concentration landscape of wind borne odors is discontinuous and consists of sporadically located patches. A pre-requisite to olfactory search is that intermittent odor patches are detected. Because of its high speed and sensitivity ${ }^{5-6}$, the olfactory organ of insects provides a unique opportunity for detection. Insect antennae have been used in the past to detect not only sex pheromones ${ }^{7}$ but also chemicals that are relevant to humans, e.g., volatile compounds emanating from cancer cells ${ }^{8}$ or toxic and illicit substances ${ }^{9-11}$. We describe here a protocol for using insect antennae on autonomous robots and present a proof of concept for tracking odor plumes to their source. The global response of olfactory neurons is recorded in situ in the form of electroantennograms (EAGs). Our experimental design, based on a whole insect preparation, allows stable recordings within a working day. In comparison, EAGs on excised antennae have a lifetime of $2 \mathrm{hr}$. A custom hardware/software interface was developed between the EAG electrodes and a robot. The measurement system resolves individual odor patches up to $10 \mathrm{~Hz}$, which exceeds the time scale of artificial chemical sensors ${ }^{12}$. The efficiency of EAG sensors for olfactory searches is further demonstrated in driving the robot toward a source of pheromone. By using identical olfactory stimuli and sensors as in real animals, our robotic platform provides a direct means for testing biological hypotheses about olfactory coding and search strategies ${ }^{13}$. It may also prove beneficial for detecting other odorants of interests by combining EAGs from different insect species in a bioelectronic nose configuration ${ }^{14}$ or using nanostructured gas sensors that mimic insect antennae ${ }^{15}$

\section{Video Link}

The video component of this article can be found at https://www.jove.com/video/51704/

\section{Introduction}

Nowadays, animals like dogs are frequently used in safety and security applications that involve the localization of chemical leaks, drugs and explosives because of their excellent smell detection capabilities ${ }^{16}$. Yet, they show behavioral variations, get tired after extensive work, and require frequent retraining as their performance decreases over time ${ }^{17}$. One way to circumvent these limitations is to replace trained dogs by olfactory robots.

Nonetheless, tracking scents and odor sources is a major challenge in robotics. In turbulent environments, the landscape of an odor plume is very heterogeneous and unsteady, and consists of sporadically located patches ${ }^{4}$. Even at moderate distances from the source, as short as few meters, detections become sporadic and only provide cues intermittently. Furthermore, local concentration gradients during detections do not generally point towards the source. Given discontinuous flow of information and limited local information when detections are made how to navigate a robot toward the source?

It is well known that insects such as male moths use chemical communication to successfully locate their mates over long distances (hundreds of meters). To do so, they adopt a stereotypical behavior ${ }^{18-20}$ : they surge upwind upon sensing an odor patch and perform an extended search called casting when odor information vanishes. This surge-casting strategy is purely reactive, i.e. actions are completely determined by current perceptions (detection and non-detection events). Yet, its implementation on olfactory robots had limited success in the past because the detection of odor patches is hampered by the slowness of artificial gas sensors.

Metal-oxide sensors used in most of the olfactory robots have response and recovery times of several tens of seconds so that they generally filter out the concentration fluctuations encountered in turbulent plumes ${ }^{21}$. In contrast, the response time of insect chemoreceptors is much shorter, e.g., the rise time of insect electroantennograms (EAGs) is less than $50 \mathrm{msec}^{22}$. Consequently, by using insect EAGs, odor pulses are 
resolved at frequencies of several $\mathrm{Hertz}^{23}$. This property makes EAG sensors well suited for the detection of odor filaments in natural plumes. We describe here a protocol for embedding insect EAGs on robots allowing for efficient olfactory searches using surge and casting strategies.

\section{Protocol}

The protocol is based on a commercially available robot (see Materials table) and male moths (Agrotis ipsilon) with their sex pheromone. Yet, it can be adapted with minor modifications to other insect species, odorants, and robots.

\section{Insects}

1. Rear larvae of Agrotis ipsilon Hufnagel (Lepidoptera: Noctuidae) on an artificial diet and maintain them in individual plastic cups until pupation at $23 \pm 1^{\circ} \mathrm{C}$ and $50 \pm 5 \%$ relative humidity as described previously ${ }^{24}$.

2. Sex pupae and keep adult males separately from females in plastic boxes. Give them free access to a $20 \%$ sucrose solution.

3. Perform experiments with males. Male moths are highly sensitive to the sex pheromone emitted by their conspecific females. In $A$. ipsilon, the major pheromone component, cis-7-dodecenyl acetate $(\mathrm{Z} 7-12: \mathrm{OAc})$ is the most active compound on the antenna.

\section{Electrophysiology}

1. Record the EAG from a whole-insect preparation, as described below (Figure 1A). Intact antennae are preferred over excised antennae because they exhibit a longer lifetime (see representative results).

2. Chlorinate two silver wires by immersion in concentrated bleach solution for $10-20$ min and rinse afterwards. This process prevents electrodes from polarizing. It has to be repeated whenever the baseline drifts during experiments or when the offset voltage between the electrodes becomes too large to be compensated by the amplifier.

3. Make glass electrodes from fire polished capillaries with an electrode puller. Fire polishing prevents scratching the chlorinated silver wire with electrodes.

4. Anesthetize a male moth with $\mathrm{CO}_{2}$ and place it inside a Styrofoam block with the head protruding from the top.

5. Tether the insect's head with painter's tape around the neck.

6. Insert a silver wire serving as reference electrode into the neck.

7. Under a stereomicroscope, immobilize one of the antennae with thin strips of painter's tape on the tip and the base.

8. Cut out the distal 2-3 segments of the antenna with surgical scissors.

9. Position the glass electrode near the cut tip of the antenna with a micro-manipulator.

10. Cut out the extremity of the glass capillary with forceps to obtain a diameter slightly larger than the cut tip of the antenna.

11. Fill the glass pipette with (in $\mathrm{mM}$ ) $6.4 \mathrm{KCl}, 340$ glucose, $10 \mathrm{Hepes}, 12 \mathrm{MgCl}_{2}, 1 \mathrm{CaCl}_{2}, 12 \mathrm{NaCl}, \mathrm{pH} 6.5$.

12. Insert the cut tip of the antenna into the glass capillary with the micromanipulator.

13. Slip the silver wire serving as the recording electrode into the largest extremity of the glass capillary.

\section{Hardware Interface}

1. Mount the whole preparation, i.e. insect-electrodes-micromanipulator, on a metal plate screwed on the top of the robot (Figure 1B). Connect the electrodes to the robot, as described below.

2. Based on previous works ${ }^{25-26}$, design a hardware interface to adapt the EAG output voltage (order $1 \mathrm{mV}$ at several $\mathrm{M} \Omega$ ) to the range appropriate for the extension board of the robot. The board accepts $0-5 \mathrm{~V}$ analog inputs and a negative voltage below $-200 \mathrm{mV}$ may cause severe damage. Follow the steps 3.2.1 to 3.2.4 to design the interface with Eagle.

1. Design a $\pm 5 \mathrm{~V}$ power supply from a $+12 \mathrm{~V}$ battery using voltage regulator $78 \mathrm{~L} 10$ (\# in Figures $2 \mathrm{~A}-2 \mathrm{C}$ ).

2. Design a headstage preamplifier (10X) based on instrumentation amplifier INA121 (\# in Figures 2A-2C).

3. Design a second-stage amplifier $(25 \mathrm{X})$ with noise filtering (first order high-pass $0.1 \mathrm{~Hz}$ filter, second order low-pass $500 \mathrm{~Hz}$ filter, notch $50 \mathrm{~Hz}$ filter) based on quad op-amps LT1079 (\# in Figures 2A-2C).

4. Design a signal conditioning stage that computes with op-amp LT1079 and diode 1 N4148 (\# in Figures 2A-2C). The total gain is 250 and the EAG output is in the range $0-5 \mathrm{~V}$ with zero being at $2.5 \mathrm{~V}$.

3. Connect the electrodes to the differential EAG inputs (\# in Figures 2A-2C). Connect the recording electrode to the inverting input of the INA121 to obtain positive EAGs.

4. Connect the EAG output (\# in Figures 2A-2C) to the 12 analog inputs of the extension board of the robot. As each input is read sequentially every millisecond, the sampling frequency is $1 \mathrm{KHz}$.

\section{Software Interface}

The main threads contain a graphical user interface (GUI), methods for signal detection and various functions for controlling the robot.

1. Write a GUI (Figure 2D) in Qt-C++ for data visualization, digital filtering (20 Hz $5^{\text {th }}$ order Butterworth low pass filter) and odor detection from the EAG. The latter can be performed in two ways: either by deconvolving the EAG with an appropriate filter (engineering approach, section 4.2), or by modeling the neural mechanisms that allow fast and reliable pheromone detection in $A$. ipsilon moths (bioinspired approach, section 4.3).

2. Deconvolution filter. The EAG is well described by a nonlinear cascade ${ }^{27}$ that consists of a static nonlinearity $w=f(x)$ and a $1^{\text {st }}$ order low-pass filter with exponential impulse function $h(t)=\exp (-t / \tau)$ for $t \geq 0$, see Figure 3A. In response to fluctuating odor concentration $x(t)$, 
the EAG output is given by the convolution integral $y(t)=h(t) * w(t)$. Deconvolution is simply obtained by the inverse of the system; that is $\widehat{W}(f)=Y(f) / H(f)$ in the frequency domain. Then, $\widehat{W}(f)=\frac{Y(f)}{\tau}+2 \pi i f Y(f)$ as the Fourier transform of the impulse response is $H(f)=1 /\left(\tau^{-1}+2 \pi i f\right)$. For signal detection, follow the steps 4.2 .1 to 4.2 .3 .

1. Perform the deconvolution process in the time domain as $\widehat{w}(t)=\frac{y(t)}{\tau}+\frac{d y}{d t}$ and $\hat{x}(t)=f^{-1}(\hat{w}(t))$, Figure 3B.

2. Approximate the nonlinearity $f^{-1}$ by a polynomial function. Fit the polynomial parameters and the time constant on input-output data pairs to minimize the mean square error between real $x(t)$ and reconstructed $\hat{x}(t)$ odor concentration.

3. Detect the pheromone hits whenever $\hat{x}(t)$ exceeds a predefined threshold.

3. Neuromorphic detector. An alternative approach to detection consists in mimicking biology. In A. ipsilon moths, central neurons receiving input from the antenna respond to the pheromone with a stereotypical firing pattern of excitation-inhibition ${ }^{13}$. A Hodgkin-Huxley type neuron model with four ionic currents $I_{\text {ion }}$ (a delayed rectifier $\mathrm{K}^{+}$current, voltage-gated $\mathrm{Na}^{+}$and $\mathrm{Ca}^{2+}$ currents, a small conductance $\mathrm{Ca}^{2+}$ dependent $\mathrm{K}^{+}$current) was previously developed to reproduce the observed physiological responses ${ }^{13}$. For signal detection, follow the steps 4.3.1 to 4.3 .3

1. Implement the neuron model as differential equations. Use the EAG signal as input current $C \frac{d V}{d t}=\sum I_{\text {ion }}+I_{L}+\bar{I}_{E A G}$ in the evolution of the membrane potential $I_{L}=g_{L}\left(E_{L}-V\right)$. Use a membrane capacitance $\mathrm{C}=22.9 \mathrm{pF}$ and a leak current given by $I_{L}=g_{L}\left(E_{L}-V\right)$ with conductance $g_{\mathrm{L}}=0.011161 \mu \mathrm{S}$ and reversal potential $\mathrm{E}_{\mathrm{L}}=-61.4 \mathrm{mV}$. The ionic currents are described by $I_{\text {ion }}=\bar{g} m^{a} h^{b}\left(E_{\text {ion }}-V\right)$ with $\frac{d m}{d t}=\left(m_{\infty}-m\right) / \tau_{m}$ and $\frac{d h}{d t}=\left(h_{\infty}-h\right) / \tau_{h}$ by where $m_{\infty}, h_{\infty}, \tau_{m}$ and $\tau_{h}$ are nonlinear functions of $\mathrm{V}$. See previous work ${ }^{13}$ for the details.

2. Simulate the neuron model in real time with Sirene by integrating the differential equations with a $4^{\text {th }}$ order Runge-Kutta method and a time step $\delta t=0.01 \mathrm{msec}$. Run the spike test $\mathrm{V}\left(t^{f}\right)>0 \mathrm{mV}$ and $\mathrm{V}\left(t^{f}-\delta t\right)<0 \mathrm{mV}$ online to obtain spike times and interspike intervals.

3. Detect the pheromone hits whenever a burst of excitation ( 3 consecutive interspike intervals $<70 \mathrm{msec}$ ) is followed by inhibition (interspike interval $\geq 350 \mathrm{msec}$ ), see Figure 3C.

\section{Representative Results}

The protocol described above was first tested with short $20 \mathrm{msec}$ pulses of pheromone (dose $1 \mu \mathrm{g}$ and $10 \mu \mathrm{g}$ ) directly puffed on the antenna. Figure 4A shows the EAGs in response to pheromone pulses. They are positive because the recording electrode was connected to the inverting input of the amplifier, as described in step 3.3. As indicated by the power spectrum, the measurement system is able to resolve pheromone pulses up to $10 \mathrm{~Hz}$. For comparison, we also tested a commercially available gas sensor. The TGS2620 is a metal oxide sensor manufactured for the detection of solvent vapors. Although the sensor presents a high sensitivity to ethanol, it was unable to follow variations in concentration (see the dashed curve in Figure 4B). The problem came from the sensor housing. The TGS2620 is commercialized with a cap that has a flameproof stainless steel gauze. The response time is slow because, in practice, it takes a certain time for the gas to diffuse through the gauze and reach the metal oxide surface. Recovery is also slow because it takes time to clean the sensor when the gas is trapped inside the cap. We therefore removed the cap and this modification improved the dynamics significantly (see the plain curve in Figure 4B). Still, there was a factor ten between the EAG and the TGS2620 $(10 \mathrm{~Hz}$ versus $1 \mathrm{~Hz})$. This comparison is nevertheless qualitative as the EAG and the TGS2620 were not tested in the same conditions.

We then assessed the stability over time of our whole-insect preparation ( $n=12$ moths) as compared to excised antennae ( $n=7$ antennae). The EAG was recorded periodically in response to pheromone stimulations (duration $500 \mathrm{msec}$, dose $1 \mu \mathrm{g}$ ). Raw EAGs (in mV) were converted to relative EAGs (percentages of initial value obtained at time $t=0$ ). Figure 5 shows very good stability of our whole-insect preparation within a working day. In contrast, EAGs recorded on isolated antennae decrease rapidly over time so that the signal falls to one half of its initial value after only $1.5 \mathrm{hr}$. This time dependence is well described by an exponential decay with a lifetime of $2 \mathrm{hr}$.

Finally, we tested the ability of the EAG robotic plateform to search for an odor source (pheromone compound Z7-12:OAc) using a reactive search strategy (Figure 6A). The search strategy combines upwind surge every time the pheromone is detected with spiral casting in the absence of detections ${ }^{28}$. The presence of pheromone is detected from the EAG by the neuromorphic detector, as described in step 4.3. Two examples of EAG recorded during the search are shown in Figure 6B. Without the odor source, the EAG remains around zero (i.e. $2.5 \mathrm{~V}$ ) with very few or no detections. The robot performs spiral casting and generally leaves the search space before reaching the target location (in $92 \%$ of the trials, $n=26$ trials, Figure $6 \mathrm{C}$ right). With the odor source (Figure $6 \mathrm{C}$ left), the EAG presents bursts of activity (detections) intertwined with periods of silence (no detections). Spiral casting mainly occurs at the plume contour (Figure $\mathbf{6 C}$ left, red line) and appears to be an efficient strategy for relocating the plume centerline when the odor is lost. In this condition, the source is generally found (success rate $=96 \%, n=44$ trials). 
A EAG preparation

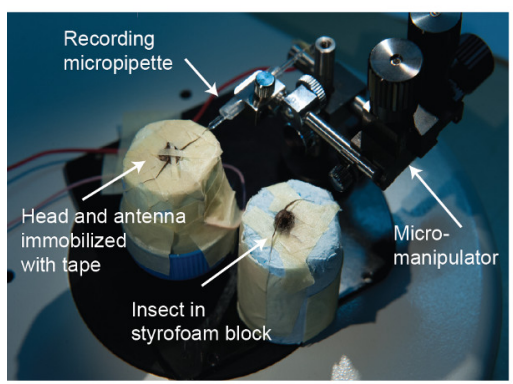

\section{B Robotic setup}

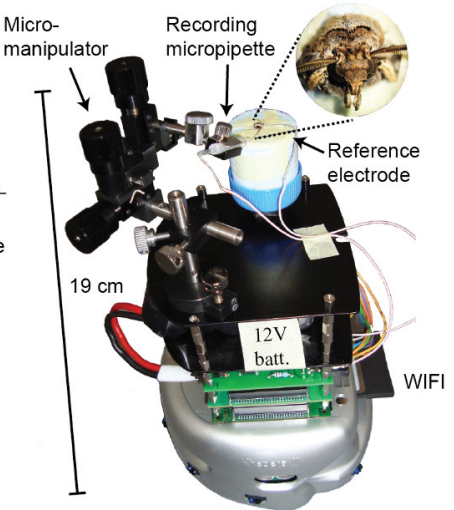

Figure 1. Whole-insect EAG preparation and robotic setup. A) The electroantennogram (EAG) is recorded from a whole-insect preparation (see text for details). B) The preparation is mounted on the robot. Please click here to view a larger version of this figure.

\section{A Schematic}
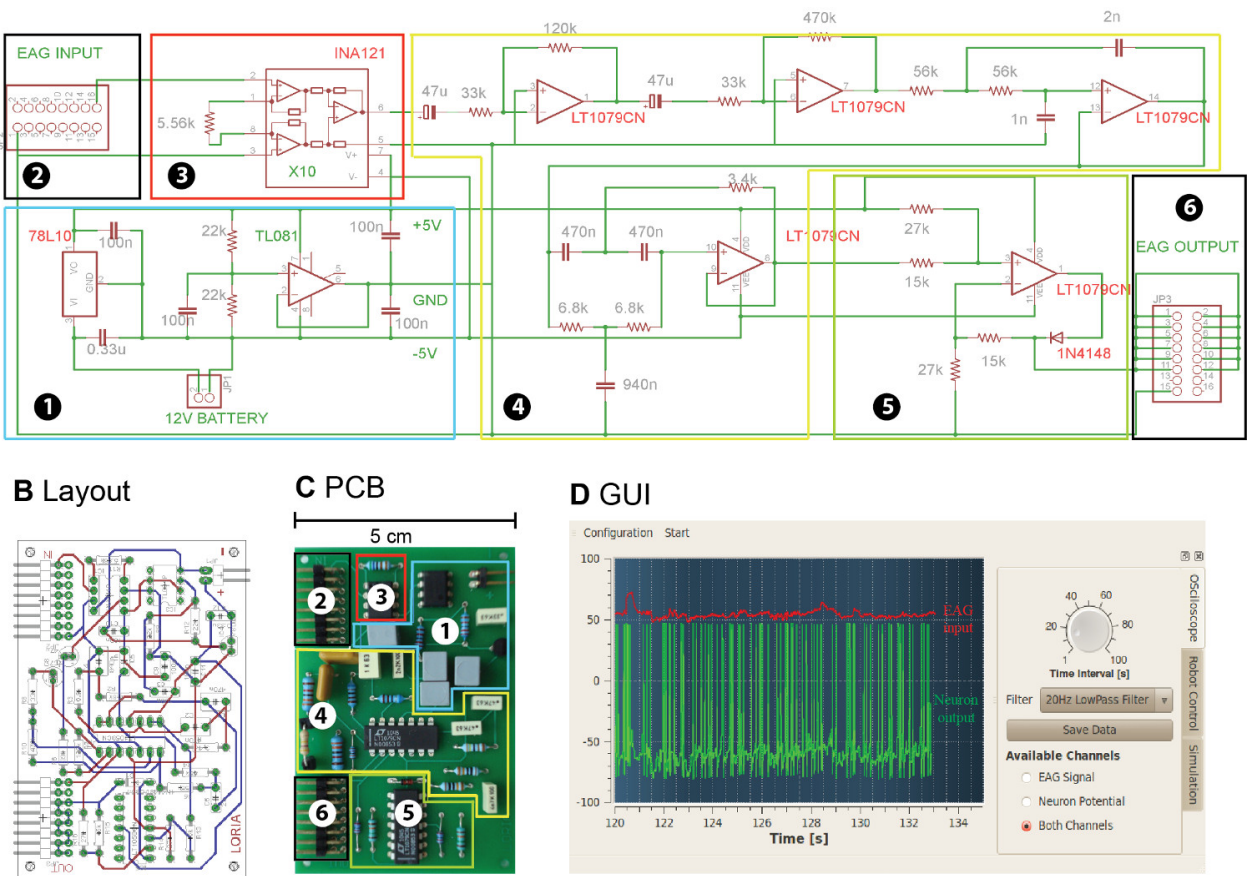

\section{GUI}

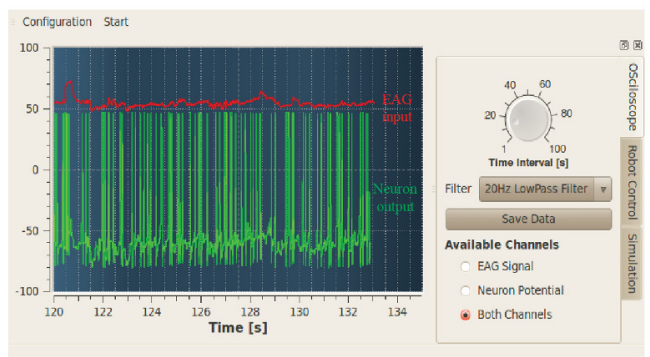

Figure 2. Hardware-software interface. A) Eagle schematic of the hardware. The circuit consists of six sections (see text for details). It allows filtering (frequency band $0.1-500 \mathrm{~Hz}$, notch at $50 \mathrm{~Hz}$ ), amplification (total gain $250 \mathrm{X}$ ) and signal conditioning in the range 0-5 V. B) Eagle layout showing lines of copper (the top is in red and the bottom in blue) and holes (in green). C) Printed circuit board (PCB) showing the discrete elements. D) Graphical user interface (GUI) written in Qt-C++ for data visualization (red trace = EAG input, green trace = neuron model output), filter design and signal detection. Please click here to view a larger version of this figure. 
A. EAG model

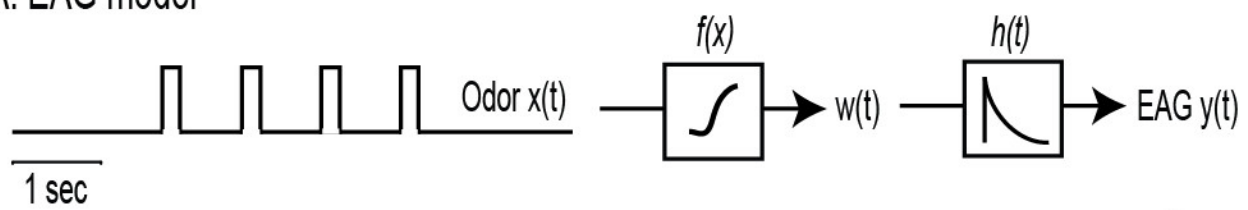

B. Deconvolution filter

odor patch

detection

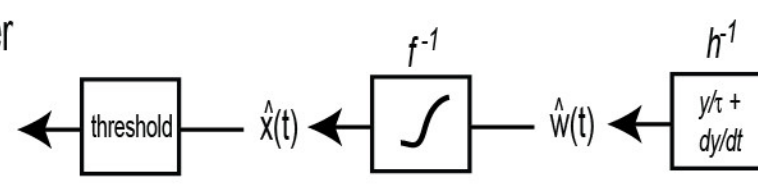

\section{Neuromorphic detector}

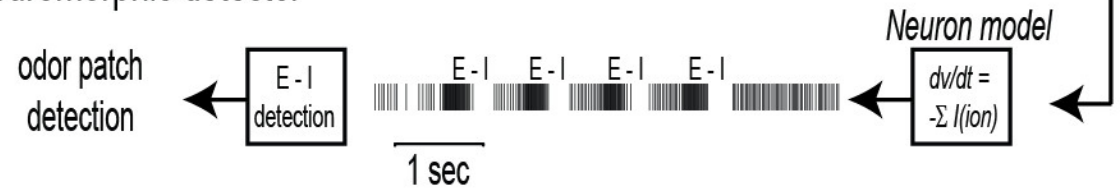

Figure 3. Signal detection from the EAG. A) Electroantennogram (EAG) model. The EAG is modeled by a nonlinear cascade ${ }^{27}$ that consists of a static nonlinearity followed by a $1^{\text {st }}$ order low pass filter with exponential impulse function $h(t)$. The EAG output is given by the convolution integral with $y(t)=h(t) * w(t)$ with $w(t)=f(x(t))$. B) Engineering approach. The deconvolution filter writes $\hat{w}(t)=\frac{y(t)}{\tau}+\frac{d y}{d t}$ and $\hat{x}(t)=f^{-1}(\widehat{w}(t))$, see text for details. Odor encounters (hits) are detected whenever $\widehat{x}(t)$ exceeds a predefined threshold. C) Bio-inspired approach. A Hodgkin-Huxley type neuron model with five internal currents (leak, $\mathrm{K}^{+}, \mathrm{Na}^{+}, \mathrm{Ca}^{2+}$ and SK) is used to reproduce the observed firing pattern of excitation-inhibition (E-I) observed experimentally ${ }^{13}$. For signal detection, the EAG signal is used as input current and hits are detected whenever a burst of excitation is followed by inhibition in the firing activity. 
A. recordings from the EAG

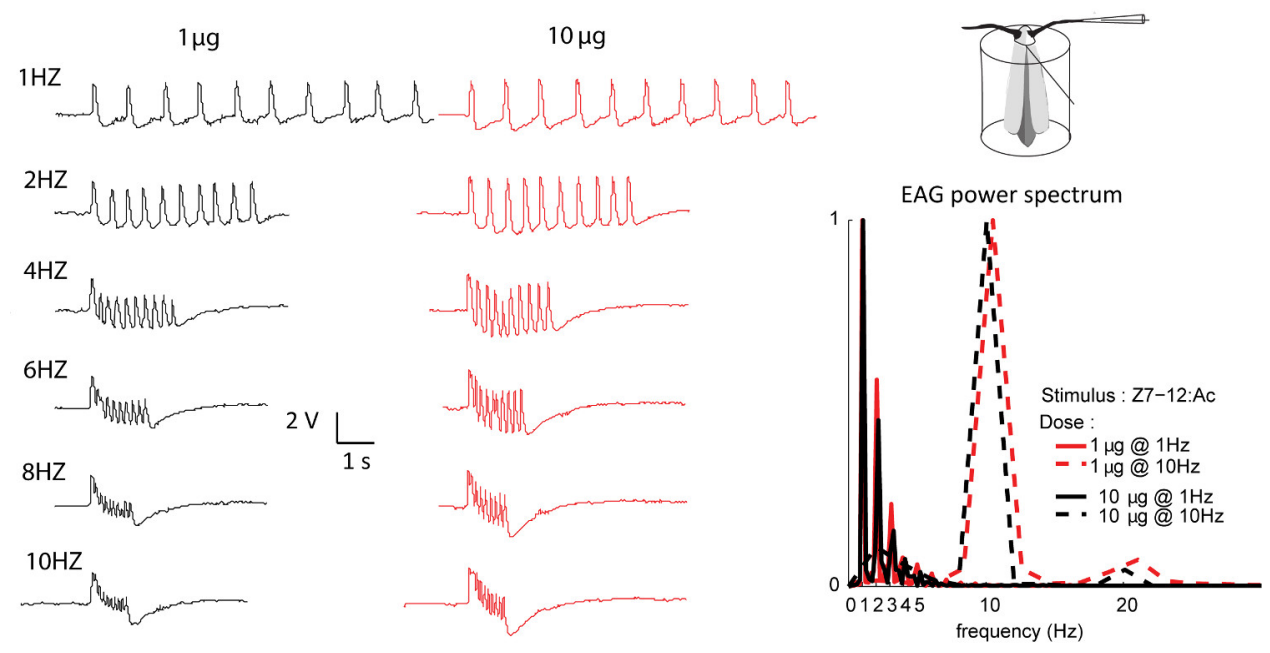

B. recordings from gas sensor TGS2620

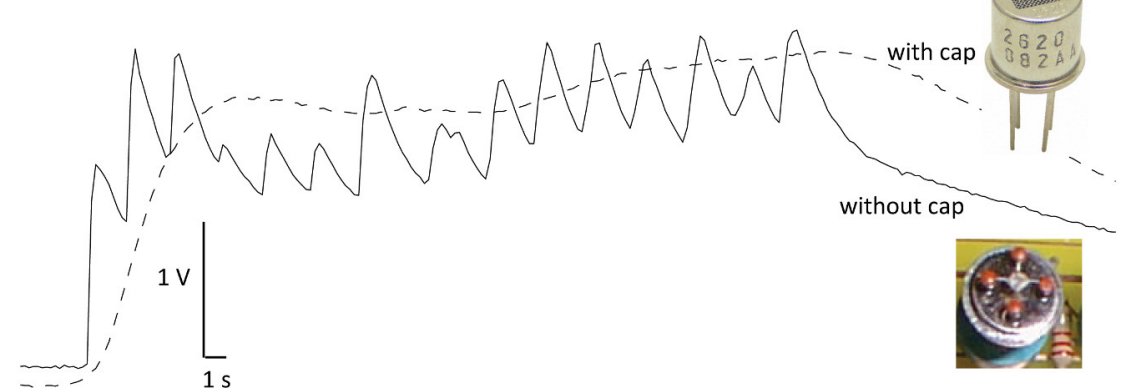

Figure 4. EAG response time. A) EAG recordings in response to $20 \mathrm{msec}$ pheromone pulses (dose $1 \mu \mathrm{g}$ and $10 \mu \mathrm{g}$ ) delivered at different rates $(1,2,4,6,8$, and 10 pulses/sec). The normalized EAG power spectrum is shown for a stimulus pulsed at 1 and $10 \mathrm{~Hz}$ (dose $1 \mu \mathrm{g}$ and $10 \mu \mathrm{g}$ ). The EAG resolves individual pulses up to $10 \mathrm{~Hz}$. B) Recordings from gas sensor TGS2620 in response to ethanol (fluctuating concentration). The dashed and plain curves are the sensor response with and without the cap, respectively. The sensor with the cap has a response time of tens of seconds and thus cannot follows the fluctuations in the gas concentration. The TGS2620 without cap resolves individual fluctuations up to $1 \mathrm{~Hz}$. Please click here to view a larger version of this figure. 


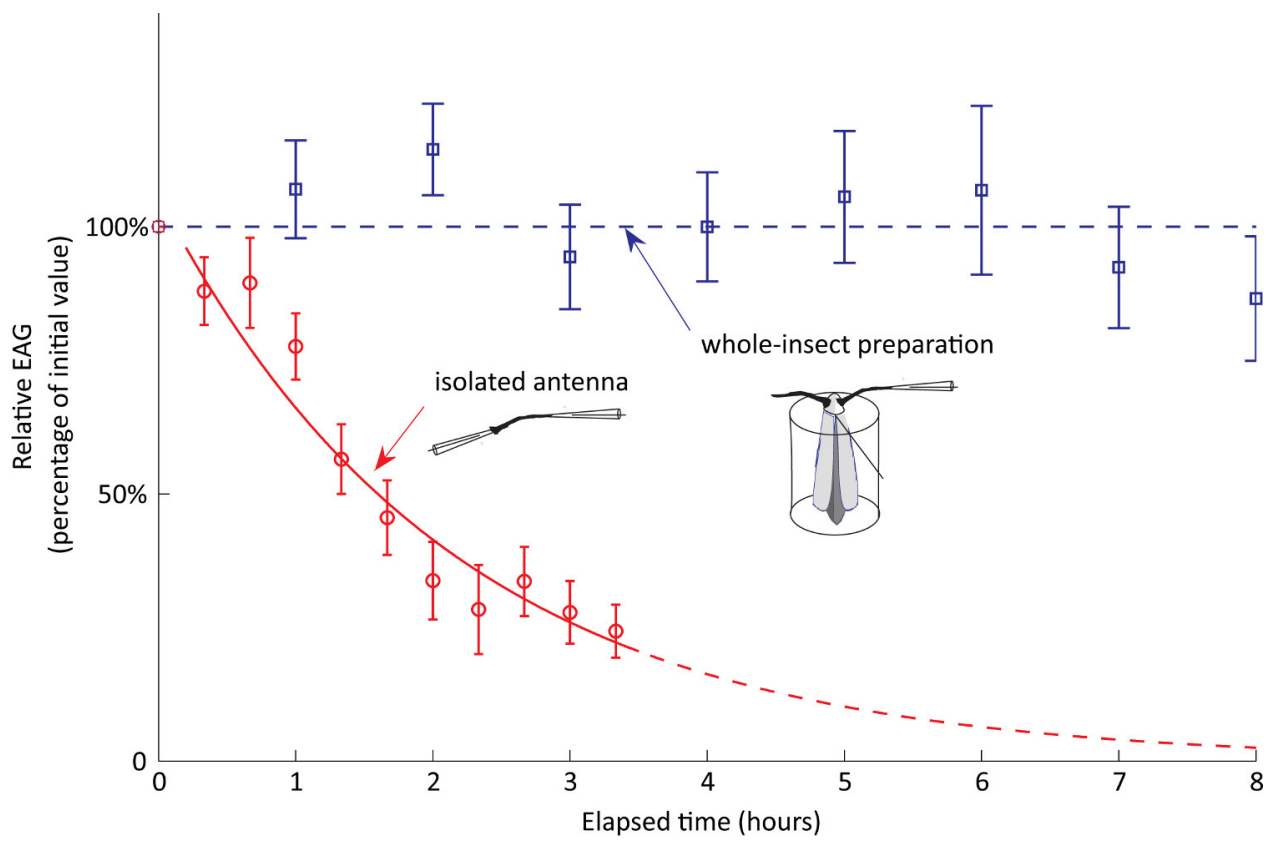

Figure 5. EAG stability (whole insect preparation vs excised antenna). The EAG was recorded every hour during $8 \mathrm{hr}$ for the whole-insect preparation ( $n=12$ moths) and every $20 \mathrm{~min}$ during $3.2 \mathrm{hr}$ for excised antennae ( $n=7$ antennae). The figure shows relative EAGs (percentages of initial value obtained at time $\mathrm{t}=0$ ). The time dependence for excised antennae is well fitted by an exponential decay with a lifetime of $2 \mathrm{hr}$ (half-life of $1.5 \mathrm{hr}$ ).

\section{A surge-casting search strategy}

\section{B typical EAG signal during search}
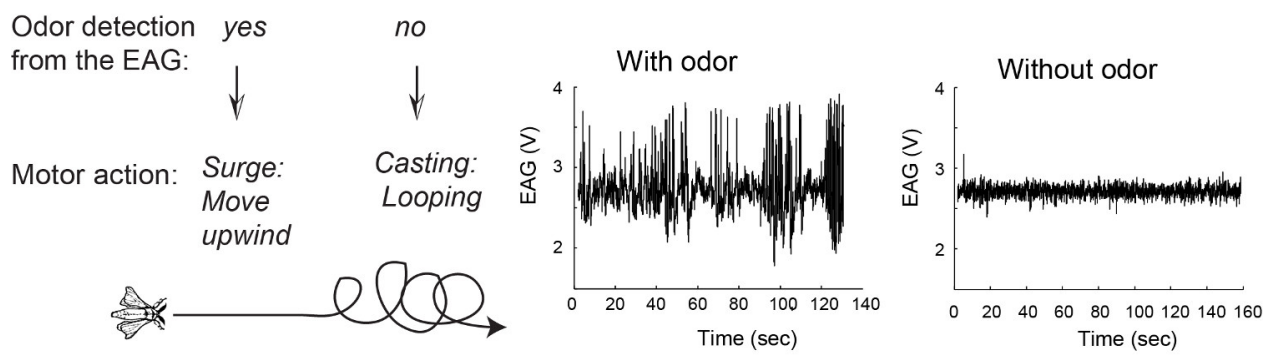

\section{Robot trajectories}

\section{With odor}

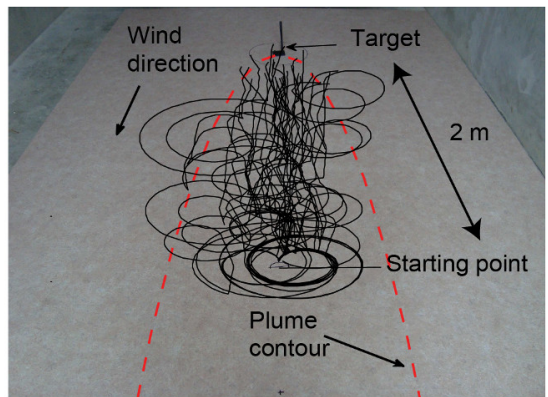

Without odor

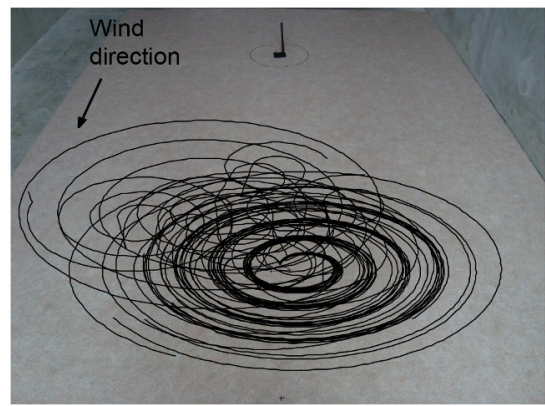

Figure 6. Robotic experiments. A) The surge-casting strategy combines upwind surge in the presence of the odor with spiral casting in its absence $^{28}$. B) Typical EAG recorded during the search while the robot is moving (with and without the odor). C) Robot trajectories with odor (n $=44$ trials) and without odor ( $n=26$ trials). The red dashed line represents the plume contour where $90 \%$ of all detections occurred during the trials. Experimental conditions : search space $=4 \mathrm{~m} \times 2.5 \mathrm{~m}$, robot's speed $=5.6 \mathrm{~cm} / \mathrm{sec}$, target $=10 \mu \mathrm{g}$ of pheromone deposited on a paper filter and replaced every 2 trials, robot initial location $=2 \mathrm{~m}$ from target, wind velocity $=0.9 \pm 0.2 \mathrm{~m} / \mathrm{sec}$ at target location. Please click here to view a larger version of this figure. 


\section{Discussion}

Almost twenty years ago, Kanzaki and his colleagues pioneered the idea of using EAGs on olfactory robots ${ }^{29-30}$. Their technique was originally based on excised antennae. Here, we recorded from intact antennae to improve the sensitivity and the lifetime of the preparation. Other studies $^{31-32}$ also noticed the superiority of whole-body preparations over isolated antennae. In our robotic experiments, we experienced stable recordings within a day. In contrast, EAGs recorded on isolated antennae have a lifetime of $2 \mathrm{hr}$ (Figure 5).

Our EAG-robotic platform was primarily developed to test biological hypotheses about olfactory coding and search strategies in insects ${ }^{13}$. Similar to central neurons receiving input from insect antennae, we connected a neuron model to a real moth antenna on a robot and performed pheromone detection based on its firing pattern. Detection and non-detection events were then used to drive the robot toward the source of pheromone. The reactive search strategy considered here was inspired by the behavioral patterns of male moths attracted by a sex pheromone. It performed well in laboratory conditions (Figure 6), allowing the localization of a low emission source (pheromone dose of $10 \mu \mathrm{g}$ in our case versus $10 \mathrm{mg}$ in previous work ${ }^{24}$ ) in a relatively large search space (initial distance from source of $2 \mathrm{~m}$ versus $10 \mathrm{~cm}$ in previous experiments $\left.{ }^{20-21}\right)$.

These robotic experiments should be considered as a proof of concept showing that insect antennae are suitable for robotic olfactory searches. Although insect antennae are known to respond to toxic gases, drugs and explosives ${ }^{9-11}$, several extensions are needed for coping with real world applications. First, a more sophisticated search method ${ }^{34-36}$ may be more efficient at distances beyond $10 \mathrm{~m}$, when the reacquisition of the plume becomes very unlikely. Second, it may be necessary to combine EAGs from different species in a bio-electronic nose configuration ${ }^{14}$ in order to detect odorants of interests. Third, stereo sensing capabilities obtained by recording from the two antennae of the same insect may prove beneficial in terms of effectiveness. Two sensors employed in parallel may indeed increase directionality. Fourth, extensions of the search strategy to collective robotic searches ${ }^{37}$ are ought to be considered for practical applications even if they are not biologically relevant in the case of moths.

\section{Disclosures}

Authors have nothing to disclose.

\section{Acknowledgements}

This work was funded by the state program Investissements d'avenir managed by ANR (grant ANR-10-BINF-05 'Pherotaxis').

\section{References}

1. Russel, R.A. Odour detection by mobile robots. World Scientific Series in Robotics and Intelligent Systems., 232 p., (1999).

2. Woodfin, R.L. Trace chemical sensing of explosives. John Wiley \& Sons. 496 p. (2006).

3. Wyatt, T.D. Pheromones and animal behavior. (second edition) Cambridge University Press; (2013).

4. Weissburg, M. The fluid dynamical context of chemosensory behavior. Biol. Bull. 198, 188-202 (2000).

5. Angioy, A.M., Desogus, A., Barbarossa, I.T., Anderson, P., Hansson, B.S. Extreme Sensitivity in an Olfactory System. Chem. Senses. 28 (4), 279-284 (2003).

6. Kaissling, K.-E. The Sensitivity of the insect nose: the example of Bombyx mori. In: Gutiérrez and Marco, editors. Biologically inspired signal processing, Springer Verlag, Berlin Heidelberg p. 45-52. (2009).

7. Sauer, A.E., Karg, G., Koch, U.T., Dekramer, J.J., Milli, R., A portable EAG system for the measurement of pheromone concentration in the field. Chem. Senses. 17 (5), 543-553, (1992).

8. Strauch, M. et al. More than apples and oranges - Detecting cancer with a fruit fly's antenna. Scientific reports. 4 (3576), 1-9 (2014).

9. Marshall, B., Warr, C.G., de Bruyne, M. Detection of Volatile Indicators of Illicit Substances by the Olfactory Receptors of Drosophila melanogaster. Chem. Senses. 35 (7), 613-625 (2010).

10. Rains, G.C., Tomberlin, J.K., Kulasiri, D. Using insect sniffing devices for detection. Trends Biotechnol. 26 (6), $288-294$ (2008).

11. King, T.L., Horine, F.M., Daly, K.C., Smith, B.H. Explosives detection with hard-wired moths. IEEE Trans. Instrumentation and Measurement. 53 (4), 1113-1118 (2004).

12. Gardner, J. and Bartlett, P. Electronic noses. Oxford University Press. 245, (1999).

13. Martinez, D. et al. Multiphasic On/Off pheromone signaling in moths as neural correlates of a search strategy. PLoS ONE. 8 (4), (2013).

14. Park, K.C., Ochieng, S.A., Zhu, J., Baker, T.C. Odour discrimination using insect electroantennogram responses from an insect antennal array. Chem. Senses. 27, 343-352, (2002).

15. Spitzer, D. et al. Bio-Inspired Nanostructured Sensor for the Detection of Ultralow Concentrations of Explosives. Angewandte Chemie. 51 (22), 5334-5338, (2012).

16. Furton, K. G, Myers, L. J. The scientific foundation and efficacy of the use of canines as chemical detectors for explosives. Talanta. 54, 487-500, (2001).

17. Williams, M., Johnston, J.M. Training and maintaining the performance of dogs (Canis familiaris) on an increasing number of odor discriminations in a controlled setting. Applied Animal Behaviour Science. 78, 55-65, (2002).

18. Murlis, J., Elkinton, J.S., Cardé, R.T. Odour plumes and how insects use them. Annu. Rev. Entomol. 37, 505-532, (1992).

19. Kaissling, K.E. Pheromone-controlled anemotaxis in moths. In: Lehler, M, editor. Orientation and communication in Arthropods. Birkhäuser Verlag, Basel/Switzerland; p. 343-374. (1997).

20. Vickers, N.J. Mechanisms of animal navigation in odor plumes. Biol. Bull. 198, 203-212 (2000). 
21. Martinez, D., Rochel, O. and Hugues, E., A biomimetic robot for tracking specific odors in turbulent plumes. Autonomous Robot, Special Issue on Mobile Robot Olfaction. 20, 185-195 (2006).

22. Szyszka, P., Stierle, J.S., Biergans, S., Galizia, C.G. The Speed of Smell: Odor-Object Segregation within Milliseconds. PLoS ONE. 7, (2012).

23. Bau, J., Justus, K.A., Loudon, C., Cardé, R.T. Electroantennographic resolution of pulsed pheromone plumes in two species of moths with bipectinate antennae. Chem Senses. 30, 771-780, (2005).

24. Barrozo, R.B., Gadenne, C., Anton, S. Switching attraction to inhibition: mating-induced reversed role of sex pheromone in an insect. J. Exp. Biol. 213, 2933-2939, (2010).

25. Land, B.R., Wyttenbach, R.A., Johnson, B.R. Tools for physiology labs: an inexpensive high-performance amplifier and electrode for extracellular recording. J. Neuroscience Methods. 106, 47-55, (2001).

26. Ortiz, L., A mobile electrophysiology board for autonomous robotics. Master thesis. The University of Arizona. (2006).

27. Justus, K.A., Cardé R.T., French A.S. Dynamic Properties of Antennal Responses to Pheromone in Two Moth Species. J. Neurophysiol. 93, 2233-2239, (2005).

28. Martinez, D., Moraud, E.M. Reactive and cognitive search strategies for olfactory robots, in Neuromorphic Olfaction, Frontiers in Neuroengineering Series, K.C. Persaud, S. Marco, A. Gutierrez-Galvez (eds.), CRC Press. (2013).

29. Kuwana, Y., Shimoyama, I., Miura, H. Steering control of a mobile robot using insect antennae. Proceedings of the IEEE/RSJ International Conference on Intelligent Robots and Systems, Pittsburgh, PA. (1995).

30. Kuwana, Y., Nagasawa, S., Shimoyama, I., Kanzaki, R. Synthesis of the pheromone-oriented behaviour of silkworm moths by a mobile robot with moth antennae as pheromone sensors. Biosensors \& Bioelectronics. 14, 195-202 (1999).

31. Myrick, A. J., Park, K. C., Hetling, J. R., and Baker, T. C. Detection and discrimination of mixed odor strands in overlapping plumes using an insect-antenna-based chemosensor system. J. Chem. Ecol. 35, 118-130 (2009).

32. Myrick, A.J., Park, K.C., Hetling, J.R., and Baker, T.C. Real-time odor discrimination using a bioelectronic sensor array based on the insect electroantennogram. Bioinspiration \& Biomimetics. 3, (2008).

33. Vickers, N.J., Christensen, T.A., Baker, T.C., Hildebrand, J.G. Odour-plume dynamics influence the brain's olfactory code. Nature. 410 , 466-470 (2001).

34. Vergassola, M., Villermaux, E., \& Shraiman, B.I. 'Infotaxis' as a strategy for searching without gradients. Nature. 445, $406-409$ (2007).

35. Martin-Moraud, E., Martinez, D. Effectiveness and robustness of robot infotaxis for searching in dilute conditions. Frontiers in neurorobotics., (2010).

36. Masson, J.-B. Olfactory searches with limited space perception, PNAS. 110, 11261-11266 (2013).

37. Masson, J.-B., Bailly Bechet, M., Vergassola, M. Chasing information to search in random environments. J. Phys. A: Math. Theor. 42, (2009). 